\title{
INFLUENCE OF HYDRATION AND CATION BINDING ON PARVALBUMIN DYNAMICS
}

\author{
J.-M. Zanotti ${ }^{1}$, J. Parello ${ }^{2}$ and M.-C. Bellissent-Funel
}

${ }^{1}$ Laboratoire Leon Brillouin (CEA-CNRS), CEA Saclay, 91191Gif-sur-Yvette Cedex, France.
${ }^{2}$ UPRES-A CNRS, Faculté de Pharmacie, 15 av. Flahault, 34060 Montpellier, France.

Hydration, internal dynamics and function [1] in proteins are intimately associated. Through its properties of $\mathrm{Ca}^{2+} / \mathrm{Mg}^{2+}$ exchange, parvalbumin a $11.5 \mathrm{kDa} \mathrm{Ca}^{2+}$ and $\mathrm{Mg}^{2+}$ binding proteins, is associated with muscle and neuron relaxation. Dynamics of parvalbumin has been previously studied by inelastic neutron scattering and solidstate ${ }^{13} \mathrm{C}$ NMR. It has been shown that the dynamics of surface charged residues in the picosecond time range, as seen by neutrons, was concomitant with the dynamics of the protein backbone, in the nanosecond time range, as seen by NMR [2]. To definitively check the consistency of our neutron and NMR data, we have extended the previous neutron scattering experiments, to new $\mathrm{Q}$ and energy resolution ranges [3].

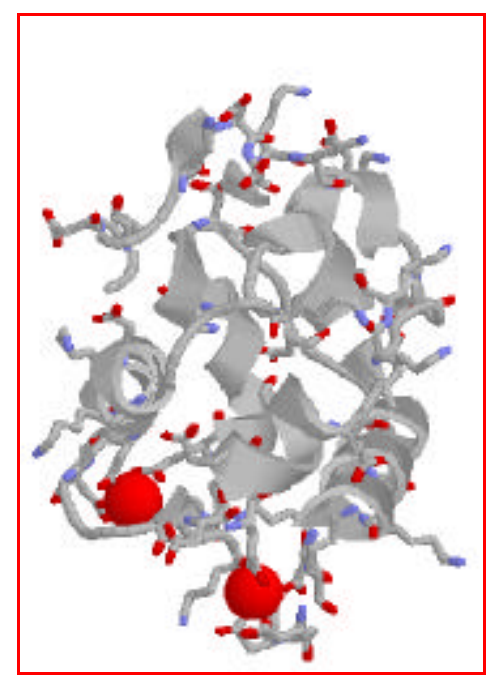

Figure 1. Ribbon representation of parvalbumin (PDB code entry : 1PVA). The two divalent ions $\mathrm{Ca}^{2+}$ or $\mathrm{Mg}^{2+}$ are symbolised by two black spheres. Charged side chains, lysines, aspartic and glutamic acids, are represented as sticks. As shown by a previous study [2], the localised relaxational dynamics of those surface residues, exposed to the solvent, give rise to the quasielastic scattering observed in the ps time range.
In particular, high $Q\left([1 ., 5.] \AA^{-1}\right)$ and high resolution $(10 \mu \mathrm{eV})$ data have been obtained on the thermal backscattering CRG IN13. The data treatment described in reference [2] has been applied. EISF obtained for each samples of $\mathrm{Ca}$ or $\mathrm{Mg}$ loaded parvalbumin hydrated at $\mathrm{h}=0.27 \mathrm{~g} 2 \mathrm{O} / \mathrm{g}$ and $h=0.72$ are presented on Fig. 2. In the present high $\mathrm{Q}$ range at high energy resolution $(10 \mu \mathrm{eV})$, a Gaussian distribution of radii has to be taken into account to properly describe the experimental EISF. We assess a fraction of mobile protons and an average confinement radius. The fraction of protons seen as immobile is strongly hydration dependent. At $\mathrm{h}=0.7$, an hydration corresponding to three water layers around the protein, on a time scale of $150 \mathrm{ps}(\mathrm{R}=10 \mu \mathrm{eV})$, the protons of almost all $(0.94 \%)$ the protein secondary structure elements undergo local relaxational dynamics. In proteins, the local (steric) environment varies strongly from side-chains to backbone (the compacity is 0.74 in the deep interior of a globular protein). Probably due to the contribution of buried protons experiencing high steric constraints, the average confinement radius decreases from $1.7 \AA$ at 15 ps [2] to $1.3 \AA$ on a longer timescale of $150 \mathrm{ps}$ (this work). Interestingly, this characteristic length is not affected by hydration.

For an incoherent system, the Q dependence of the long-time tail of the intermediate scattering function, $\mathrm{I}(\mathrm{Q}, \mathrm{t}=\infty) / \mathrm{I}(\mathrm{Q}, \mathrm{t}=0)$, is the form factor of a confinement volume: the EISF. As shown in Fig.1, at a time scale of $150 \mathrm{ps,}$, accessible with the resolution of $10 \mu \mathrm{eVof}$ IN13, NSE and backscattering experiments show some very good agreement. Nevertheless, the comparison between IN13 CRG and IN11 data, showing a significant decrease of the intermediate scattering function beneath the value of extrapolated EISF, suggest that, on a time scale of few ns, new relaxational modes appear. 


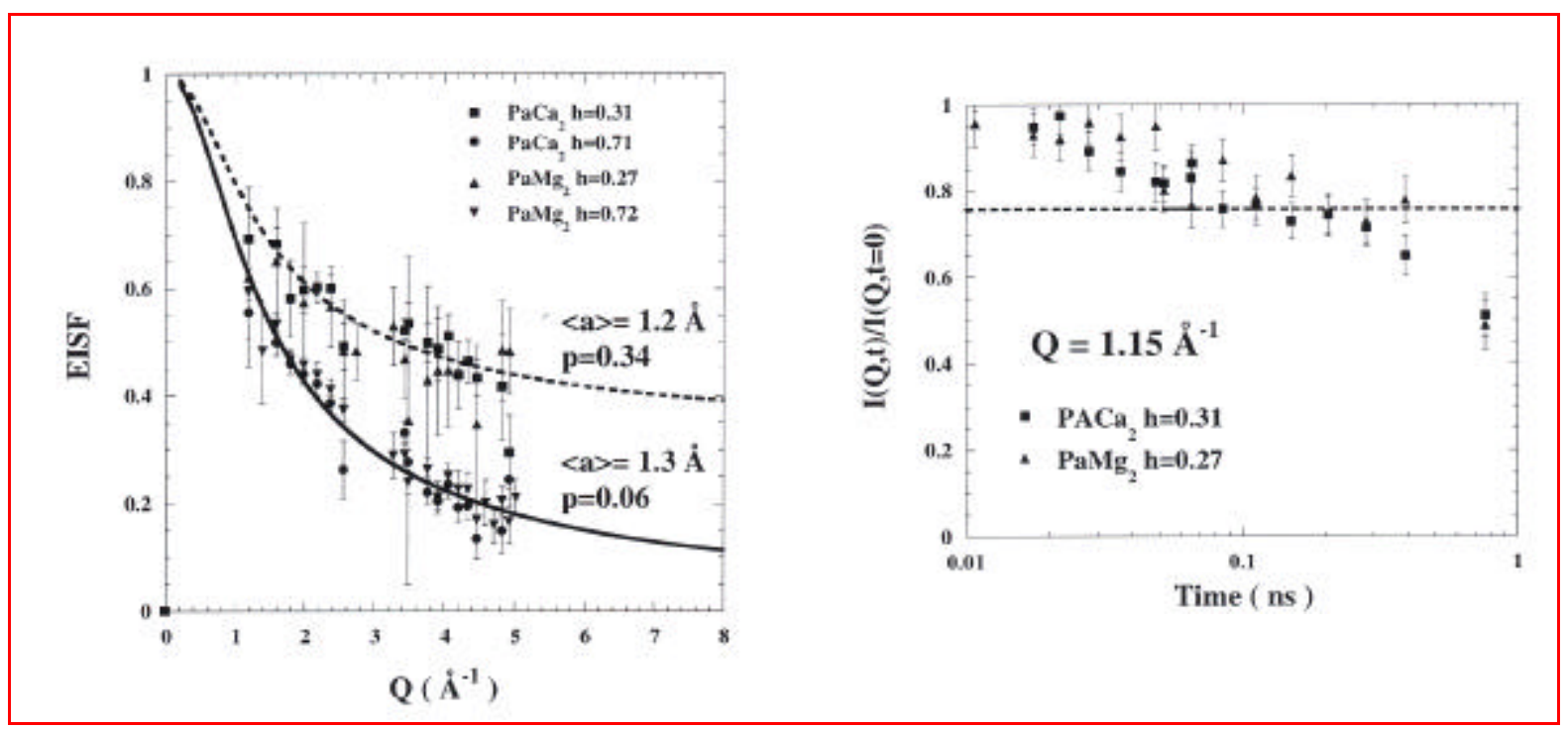

Figure 2. Left) Elastic Incoherent Structure Factor (EISF) of parvalbumin as a function of hydration and divalent ions. Measurements have been performed on IN13 at T=278 K. Dotted and full lines are the fit, for low and high hydration respectively, considering a fraction (1-p) of particles diffusing inside spherical confinement volumes with Gaussian distributions of radii. At each hydration the fitted fraction of immobile protons, $p$, and the averaged radius $<a>$ are specified. Right: $\mathrm{PaCa}_{2}$ and $\mathrm{PaMg}_{2}$ NSE spectrum obtained on IN11C (ILL) at T=298 K. The dotted line indicates the level of the EISF deduced at $\mathrm{Q}=1.15 \AA^{-1}$, from the parameters extracted from IN13 experimental EISF (Fig. 1 left). At a time scale of $150 \mathrm{ps}$, accessible with the resolution of $10 \mu \mathrm{eV}$ of IN13, NSE and backscattering experiments show very good agreement. At longer time, the decrease of the intermediate scattering function beneath the value of extrapolated EISF, suggests that relaxational modes, not seen at a timescale of few hundred of ps, appear on the nanosecond time scale.

\section{Conclusion:}

No difference has been detected in the dynamics of the calcic and magnesic forms of parvalbumin from ps to ns. The question remains whether there are really no dynamical changes or whether these changes are so local, that they are blurred out by the averaging over all the protein protons.

When increasing energy resolution the strong decrease, nearly to 0 , of the fraction of protons seen as immobile, has confirmed that it is not only the polar side-chains at the surface of the protein_which are primarily affected during the early steps of hydration. Internal non-polar sidechains and backbone are also affected. These results and others presented in [3] confirm our previous NMR and neutron scattering results that hydration acts on the dynamics of the protein at both local and global levels. This is probably essential for the biological function of the protein.

\section{References}

[1] J.A. Rupley and G. Careri, Adv. Protein Chem., 41, 37 (1991).

[2] J.-M. Zanotti, M.-C. Bellissent-Funel and J. Parello, Biophys. J., 76, 2930(1999).

[3] J.-M. Zanotti, M.-C. Bellissent-Funel and J. Parello, Applied Physics A, 74 : S1277-S1279 (2002). 